\title{
Sodium Chloride Crystallization by Electric Discharge in Brine
}

\author{
Jairo Breno Francisco de Oliveira Barauna ${ }^{a} *$, Camila Senna Pereira ${ }^{b}$, Ingrid Azevedo Gonçalves ${ }^{b}$, \\ Jussier de Oliveira Vitoriano ${ }^{c}$, Clodomiro Alves Junior ${ }^{d}$ \\ ${ }^{a}$ Centro de Ciências Exatas e Naturais (CCEN), Universidade Federal Rural do Semi-Árido (UFERSA), \\ Mossoró, RN, Brasil \\ ${ }^{b}$ Centro de Engenharias (CE), Universidade Federal Rural do Semi-Árido (UFERSA), \\ Mossoró, RN, Brasil \\ cPrograma de Pós-graduação em Engenharia Mecânica (PPGEM), Universidade Federal do \\ Rio Grande do Norte (UFRN), Natal, RN, Brasil \\ ${ }^{d}$ Centro de Ciências Exatas e Naturais (CCEN), Universidade Federal Rural do Semi-Árido (UFERSA),
} Mossoró, RN, Brasil

Received: January 17, 2017; Revised: April 04, 2017; Accepted: June 03, 2017

\begin{abstract}
Electrical discharges in liquids are currently used to synthesize nanoparticles. The plasma-liquid interaction is complex, where parameters such as electric field, ion charges and other species present are important. In order to understand the mechanism of crystallization, mother liquor (saturated solution of $\mathrm{NaCl}, \mathrm{MgCl}_{2}$ and $\mathrm{KCl}$ ) was used to study the effectiveness and selectivity of the crystallization caused by the application of plasma. Discharge was applied with the solution at room temperature or during cooling. X-Ray Diffraction (XRD), Scanning Electron Microscopy (SEM) and chemical analysis was used to evaluate the structure and composition effects on crystal structure and composition. It was observed that the discharge induces selectively crystallization, and when compared to crystals obtained conventionally by cooling, were smaller and more uniform. We therefore confirm that plasma in liquid can also be used to selectively crystallize materials in atmospheric pressure, where the sizes of the crystals must be dependent on the parameters used.
\end{abstract}

Keywords: Electric Discharge, plasma-liquid interaction, crystallization, sodium chloride, brine

\section{Introduction}

Plasma-liquid interactions is a field that gained highlights in the last years. That is justified by the low cost, ease of implementation and versatility ${ }^{1}$. Using this interactions, it is possible, for example, the synthesis of nanoparticles ${ }^{2}$, polymers $^{3}$, surface disinfection ${ }^{4}$, and effluent treatment ${ }^{5}$. This processes are driven by many mechanisms generated in the plasma-liquid interface: solvated electrons, gaseous ions impacting the surface of the liquid, reactive species, $\mathrm{UV}$ radiation and strong electric fields ${ }^{6,7}$.

Crystallization from saturated solutions is driven by changes in the free energy ${ }^{8}$, and that is the reason why it is sensible to processes involving electric discharges in liquids. In fact, some of the physical and chemical processes avaible in the plasma-liquid interface were already studied to induce or assist crystallization, as $\mathrm{UV}^{9}$, solvated electrons ${ }^{10}$ and electric fields ${ }^{11,12}$.

The production of table salt from the solar evaporation of seawater discharges, generally at the ocean, a concentrated saline effluent ${ }^{13}$. It happens mostly because there are not viable alternatives to separate the sodium chloride from the other compounds in this brine, as magnesium chloride, potassium chloride, and sodium bromide ${ }^{14}$.
The residual volume of that evaporation, corresponds to nearly $20 \%$ of the original input and is rich in many ions as Sodium, Potassium, Magnesium, Bromide, Chloride and Sulfate ${ }^{15}$. That complex composition means that when a phase change is favorable, the crystallization will occur to the compound which is more sensible to the variations in the energy ${ }^{16}$.

To modify or control the compounds obtained by crystallization, it is needed to modify the conditions on which they are crystallized, and two of the main features of well controlled crystals are the size distribution and phases formed. To obtain better control on that variables, new alternatives are being proposed ${ }^{17}$.

Plasma-liquid interactions have potential to be useful in that area, since the electric discharge transfers charge as a solid electrode, but without any surface to adsorption ${ }^{7}$. It means that is possible to produce compounds in the liquid surface just above the discharge, and they will not be adhered to any solid surface as a film.

The most common use of this features in the scientific field is to produce nanoparticles, but it was also recently discovered that a plasma irradiated culture medium could crystallize calcium oxalate ${ }^{18}$. 
However, this work is the first effort to better understand which differences an electric discharge would produce in the crystallization of salts from a saturated solution. And for that, we generated a discharge in the surface of a saturated brine from de table salt industry and analyzed if this induces crystallization. Also, we compared the solids obtained by the plasma-liquid interaction, and naturally, in a cooling crystallization.

\section{Materials and Methods}

Samples of saturated brine (density 1,28 kg/l) were obtained in the solar salt industry Salinor - Salinas do Nordeste Ltda, Areia Branca - RN. They were collected in the crystallization tank, and stored in plastic bottles, under room temperature $\left(25^{\circ} \mathrm{C}\right)$. Two experiments were made to evaluate the effect of the electric discharge in the crystallization. In the Experiment 1 (Fig. 1), a discharge was started between a $20 \mathrm{ml}$ brine sample in a petri dish, and a simple stainless-steel electrode (any stainless-steel is suitable for that application, because there is not high temperatures, or corrosion problems, as it is used as cathode), with a thin tip of $2 \mathrm{~mm}$ and $5 \mathrm{~mm}$ away from the solution, for 5 minutes. It was evaluated if the discharge itself would be enough to induce a crystallization. The solution was weighted before and after the discharge and the solids obtained were filtered using an $8 \mu \mathrm{m}$ paper filter, and then dried in the drying oven $\left(60^{\circ} \mathrm{C}\right)$.

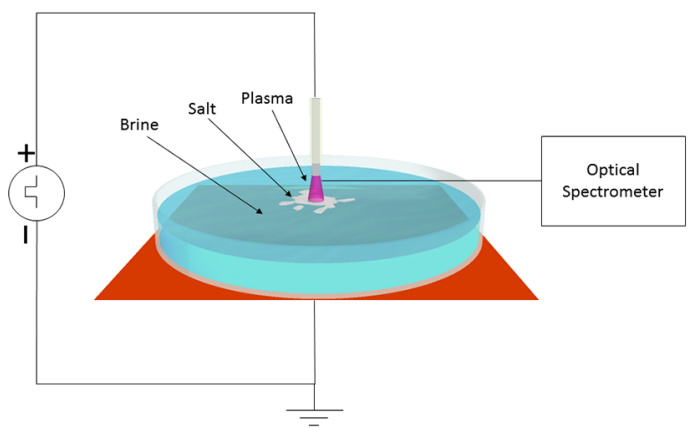

Figure 1. Apparatus to start the discharge on the surface of the brine.

The experiment 2 (Fig. 2) was made using two beckers containing $200 \mathrm{ml}$ of brine, that were heated to $60^{\circ} \mathrm{C}$ and evaporated $30 \%$ of its initial volume, to raise the concentration. From each Becker, a sample of $20 \mathrm{ml}$ was obtained, and both were put to cool in identical petri dishes until they reached room temperature. In one of the samples a discharge was initiated in the same way as in the Experiment 1, during the first 5 minutes of cooling, and the other one was used as control. Both samples were weighed every minute, and the temperature was measured every 5 minutes until the end of the cooling, when they reached the room temperature $\left(25^{\circ} \mathrm{C}\right)$. So, it was possible to plot a graph with the mass loss and temperature over time. The experiment 2 was used to compare the crystals obtained from a conventional cooling method with the ones under the discharge effect.

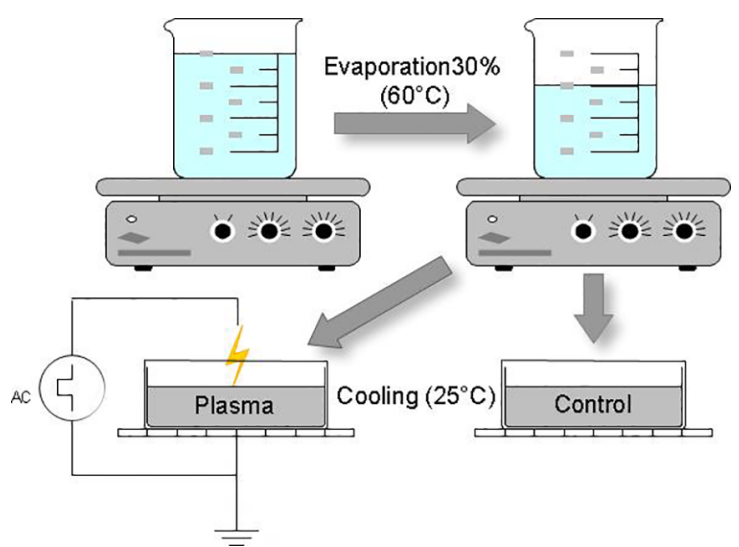

Figure 2. Experiment 2 scheme.

To power the discharge, a pulsed current power supply $(10 \mathrm{kV}$ and $990 \mathrm{~Hz}$ ) was used. The upper electrode was the cathode, and was in a glass tube, with its tip exposed, $5 \mathrm{~mm}$ away from the solution. The earth electrode was a copper plate, under the petri dish. No additional gas was used, so the discharge was conducted in the atmospheric gas. During both experiments, the temperature was monitored using an Infrared Thermometer Minipa MT-350. During the Experiment 1, an Optical Spectrophotometer Ocean Optics USB4000 in the beginning of the discharge and after 5 minutes. The solids filtered were analyzed using a XRD Shimadzu model 6000, $\mathrm{CuK} \alpha$ radiation. Sodium and potassium were determined using a Flame Spectrometer DM-62. Magnesium, chloride and sulfate were determined by titrations. Images of the crystals were taken using a Scanning Electron Microscopy (SEM). The size distribution of the crystals obtained in the Experiment 2 were also analyzed using the software ImageJ.

\section{Results and Discussion}

\subsection{Experiment 1}

Immediately after the beginning of the discharge, it was observed that crystals start to form under the plasma, spreading across the liquid on the electrode's surroundings, as the time passes (Fig. 3). There was no significant temperature increment, however, it was registered a mass loss of $1 \%$ after the 5-minute treatment.

That can be explained because the generated discharge can be assumed as a cold atmospheric discharge, where the electrons have high temperature but do not transfer that temperature to the heavier species $^{19}$. On the other hand, there is a mass reduction, that is not caused by the conventional evaporation, since there is not a significant temperature rising. So, to explain that, it is proposed that a nonequilibrium evaporation is happening, which was already visualized in the interaction between electric discharges and aqueous solutions ${ }^{20}$. It is caused mainly by the impact of ions coming from the plasma, hitting the liquid surface driven 


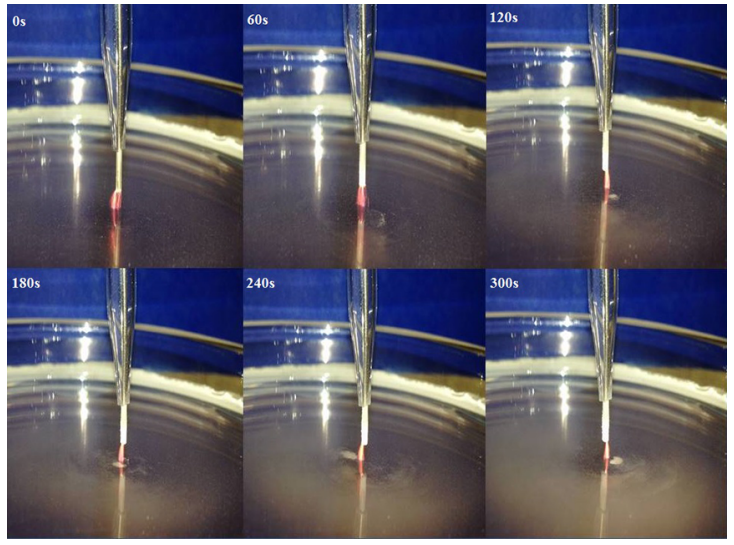

Figure 3. Crystals forming under the electric discharge effect.

by the electric field. That sputtering effect can eject atoms and molecules from the liquid into the gaseous phase and can also be helped by the transport of water attached to ions attracted by the electric field ${ }^{21}$. If those atoms and molecules are being ejected from the solution, then some of them may be seem in the plasma-phase, as ionized or excited species.

So, an evidence-based approach was made by Optical Emission Spectroscopy, to verify what species were present in the discharge zone. And indeed, it was possible to identify Sodium (Na I at 589,59240 nm) and Chloride (ClI at 767,24 and $771,76 \mathrm{~nm}$ ) emissions, clearly coming from the solution. In addition, it was possible to see reactive $\mathrm{OH}$ radicals (coming from water molecules, at $315 \mathrm{~nm}$ ), nitrogen and oxygen species from the atmospheric gas (N II at 315.93 $\mathrm{nm}, \mathrm{NO}$ I at $338,64 \mathrm{~nm}, \mathrm{~N}$ II at $359,36 \mathrm{~nm}, \mathrm{~N}$ I at 379,65 ; and $\mathrm{O}$ II at 376,$24 ; 397,32$ and $407,88 \mathrm{~nm})^{22}$.

After 5 minutes of discharge, the second OES analysis shows only sodium and chloride, which suppressed the other peaks. That means that the transport of elements from the solution to the gas phase is time-dependent, agreeing with earlier observations ${ }^{23,24}$ (Fig. 4).

To explain that difference on the scope of this work, it can be assumed that while the discharge time passes and crystals are formed under the discharge, a compositional gradient with more sodium and chloride is established, by the migration of those ions to the discharge zone, by the action of the electric field. Once they are ejected to the gaseous phase, because they are bigger ions than the nitrogen and oxygen from the atmosphere ${ }^{25}$, they are more prone to impact ionization, that causes the sodium and chloride intensities to be higher.

The chemical analyses (displayed on the pizza graphs), corresponds to the percentage of the element in the solids obtained, showing that there was a minor potassium impurity. However, the XRD analysis showed only peaks of $\mathrm{NaCl}$ (Fig. 5), that is acceptable because the percentage of potassium is very low. Also, the composition is correlated with the brine density, that indicates a saturation where the

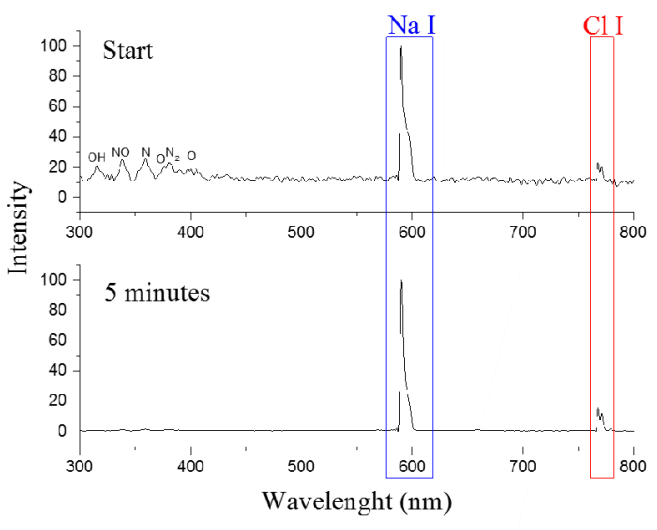

Figure 4. Optic Emission Spectroscopy from the discharge zone, in the beginning of the discharge ad after 5 minutes.

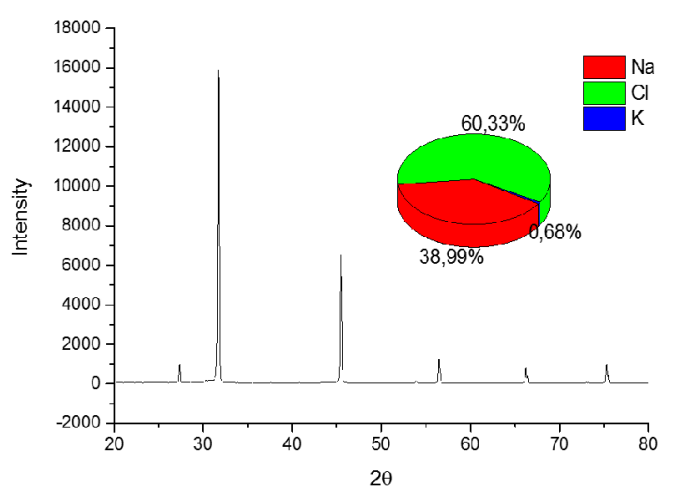

Figure 5. XRD pattern and chemical composition of the crystals obtained in experiment 1 .

sodium chloride is precipitating ${ }^{15}$. So the discharge instantly formed the compound that was already saturated.

However, at the brine concentration, it would be expected the coprecipitation of magnesium sulfate with the sodium chloride ${ }^{14,26,27}$. That was not observed on experiment 1 , which is probably related to the low mobility of the magnesium and sulfate ions in comparison with the sodium, potassium and chloride ions ${ }^{28}$. The last ones, then, get faster to the discharge site and are involved in the crystallization. Also, the SEM images (Fig. 6) showed that cubic crystals were obtained, which is in agreement with the pattern of sodium chloride crystals ${ }^{14}$.

\subsection{Experiment 2}

In the experiment 2 , the sample that interacted with the discharge behaves like in the experiment 1 , and crystals are readily formed at the point where the discharge is in contact with the liquid. The control sample crystallizes in a more dispersed layout (Fig. 7).

That difference is caused by the orientation promoted by the electric field, that makes possible to crystallize in a 


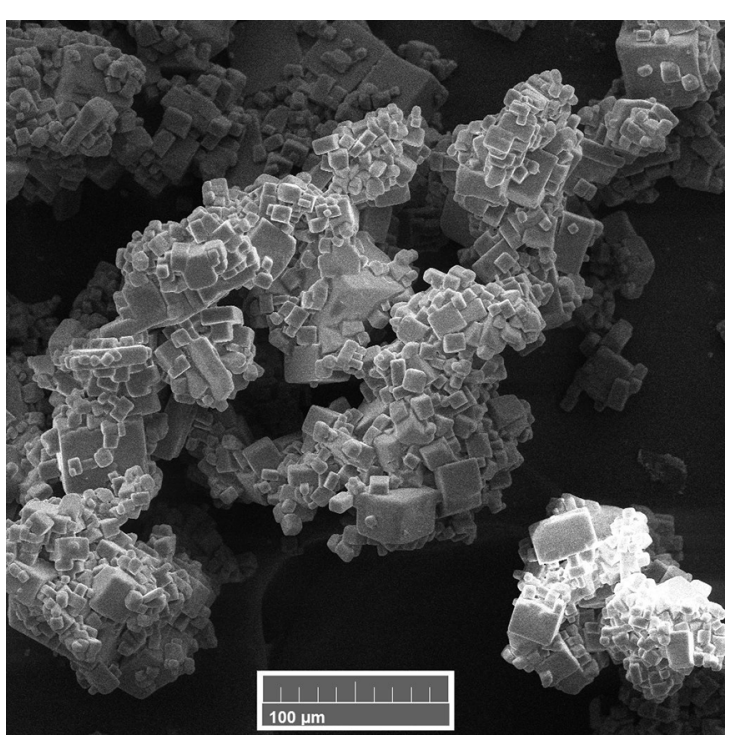

Figure 6. Scanning electron microscopy of the crystals obtained in experiment 1.

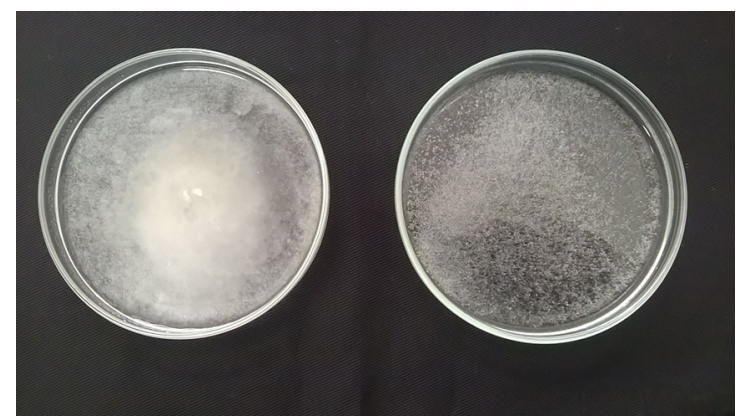

Figure 7. Samples (Left: with discharge, Right: control) after 15 minutes of cooling.

specific point on the liquid surface. So, the electric discharge crystallization allows the formation of crystals that are not in contact with solid surfaces, and in a place with different composition and energy.

Also, it has been noticed that the discharge did not changed the cooling profile of the sample when compared to the control, but again, there is a mass reduction related to the discharge (Fig. 8), and as proved in the experiment 1, it can be assumed as a nonequilibrium evaporation.

The XRD patterns were normalized for better comparison, and showed only sodium chloride peaks in both samples, but with different intensities. It is possible to notice that, with the discharge, the patterns were more coincident with the standard pattern of sodium chloride ${ }^{29}$. The control, on the other hand, had two peaks which were much higher than the others (Fig. 9).

Those differences are due to the movement of the particles driven by the electric field, which also reduced the size of the crystals, and facilitated the visualization of more crystalline planes in the XRD. Actually, the SEM images proved that assumption (Fig. 10).

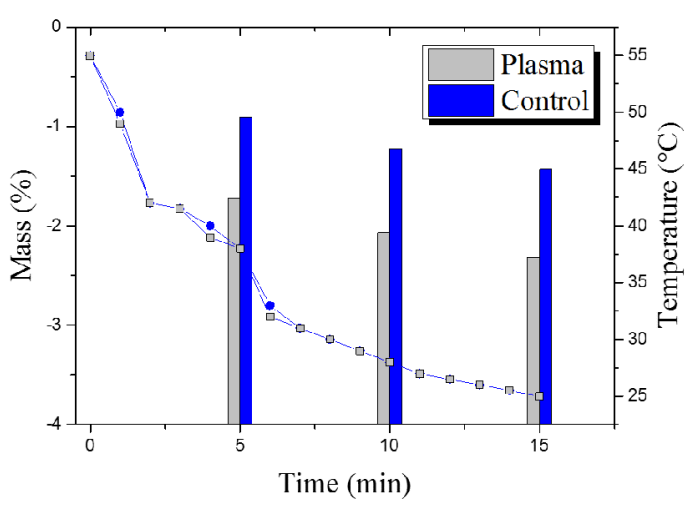

Figure 8. Mass loss (bars) and temperature variation (lines) during experiment 2 .

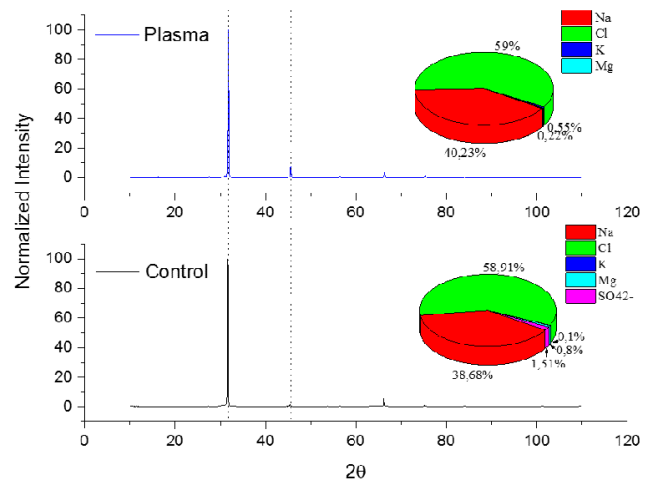

Figure 9. XRD Patterns and chemical composition, experiment 2.

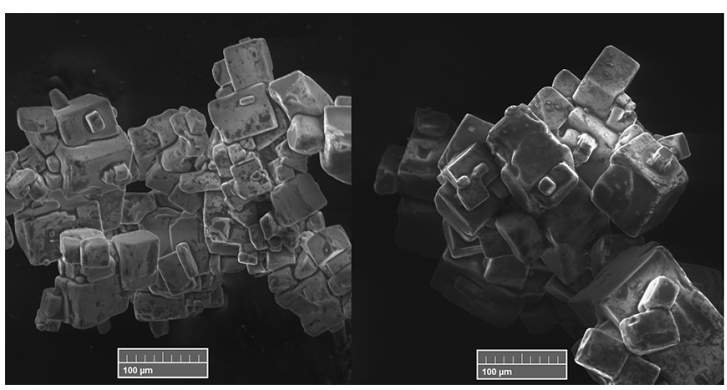

Figure 10. SEM sample with the discharge interaction (left) and control (right).

The image analysis showed that the medium size of the biggest side of the crystals with the discharge is almost half of the ones in the control sample and with a size distribution localized mostly between 10 and $30 \mu \mathrm{m}$ (Fig. 11 and Table 1).

The compositional analysis showed that the crystals obtained using the discharge were a sodium chloride with less other compounds (Fig. 9), and that is caused by the same observed in the experiment 1 . That assumption is more accepted by the rising in the potassium on the sample with the discharge. That is expected if we consider that the mobility plays and important role in this technique, since potassium 


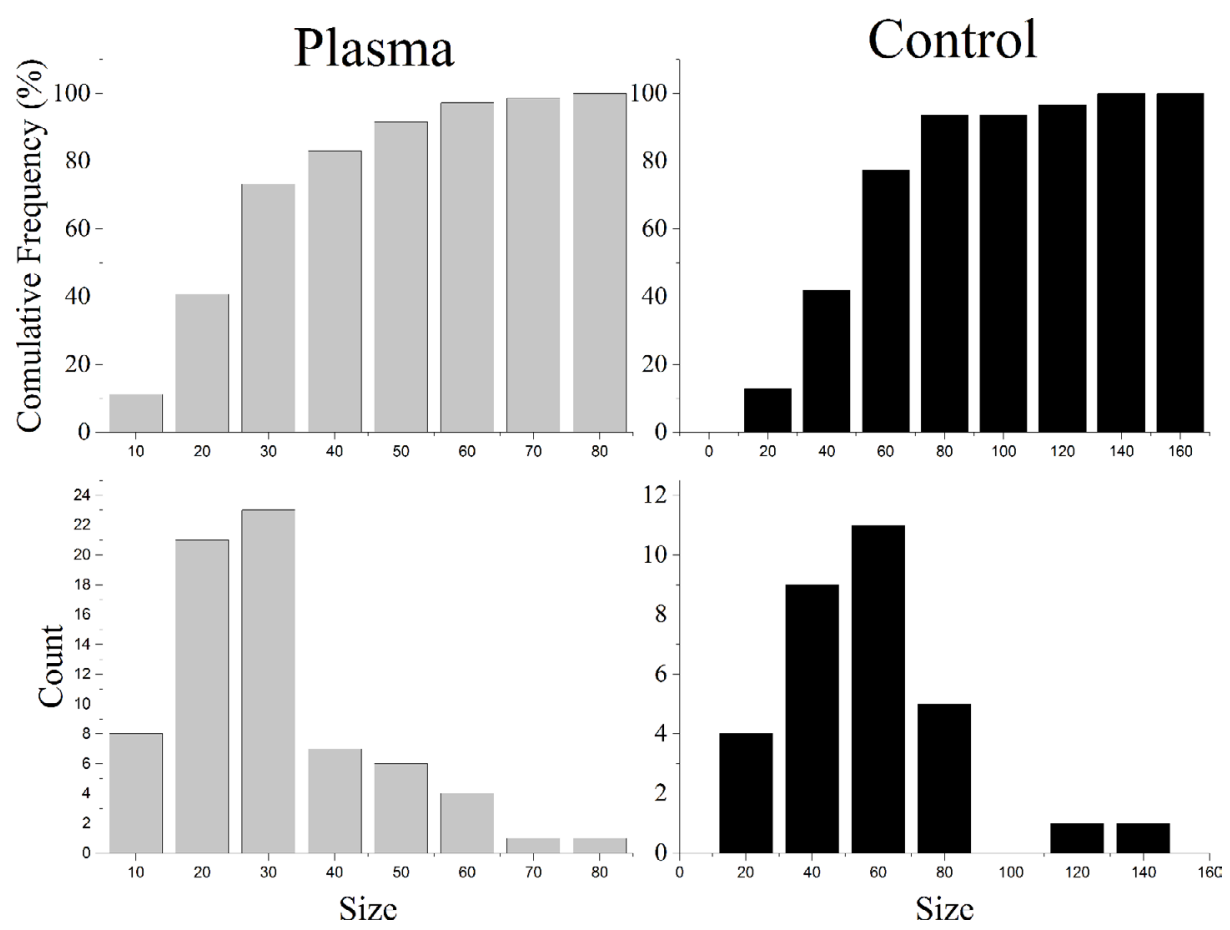

Figure 11. ImageJ analysis with crystal size distribution and quantity of crystals, (size in $\mu \mathrm{m}$ ).

Table 1. Data from the image analysis (values in $\mu \mathrm{m}$ ).

\begin{tabular}{ccccccc}
\hline Sample & Mean & Standard Deviation & Variance & Min. & Med. & Max. \\
\hline Plasma & 26,1688 & 15,06065 & 226,82303 & 5,904 & 23,484 & 78,313 \\
Control & 47,25413 & 26,31713 & 692,59148 & 13,771 & 44,693 & 139,756 \\
\hline
\end{tabular}

ionic mobility is higher than sodium and magnesium ${ }^{28}$. It is not a significant part of the solids because there is relatively less potassium at the solution than sodium. One more clue to indicate the dependence of the ionic mobility is that no sulfate (a slow ion) was confirmed in the sample with the discharge, while it appeared in the control sample.

\section{Conclusion}

Applying an electric discharge on the surface of a saturated brine can induce crystal formation. The solids obtained by this technique are different from the ones obtained conventionally by a cooling crystallization, in size and composition. Here we propose that those changes are driven by the orientation and movement of ions and molecules under the electric field and that there is the tendency to produce compounds that have faster ions in solution.

\section{Acknowledgments}

We thank CAPES and CNPq development agencies for financial support.

\section{References}

1. Chen Q, Li J, Li Y. A review of plasma-liquid interactions for nanomaterial synthesis. Journal of Physics D: Applied Physics. 2015;48(42):424005.

2. Lee SW, Liang D, Gao XPA, Sankaran RM. Direct Writing of Metal Nanoparticles by Localized Plasma Electrochemical Reduction of Metal Cations in Polymer Films. Advanced Functional Materials. 2011;21(11):2155-2161.

3. Senthilnathan J, Weng CC, Liao JD, Yoshimura M. Submerged Liquid Plasma for the Synthesis of Unconventional Nitrogen Polymers. Scientific Reports. 2013;3:2414.

4. Traylor MJ, Pavlovich MJ, Karim S, Hait P, Sakiyama Y, Clark DS, et al. Long-term antibacterial efficacy of air plasma-activated water. Journal of Physics D: Applied Physics. 2011;44(47):472001.

5. Magureanu M, Piroi D, Mandache NB, David V, Medvedovici A, Parvulescu VI. Degradation of pharmaceutical compound pentoxifylline in water by non-thermal plasma treatment. Water Research. 2010;44(11):3445-3453.

6. Mussard MDVS, Foucher E, Rousseau A. Charge and energy transferred from a plasma jet to liquid and dielectric surfaces. Journal of Physics D: Applied Physics. 2015;48(42):424003.

7. Akolkar R, Sankaran RM. Charge transfer processes at the interface between plasmas and liquids. Journal of Vacuum 
Science \& Technology A: Vacuum, Surfaces, and Films. 2013;31(5):050811.

8. Gibbs JW. On the equilibrium of heterogeneous substances. American Journal of Science. 1878;16(96):441-458.

9. Kim DY, Shim MS, Kim CH, Yi J. UV crystallization of poly-si using a $\mathrm{CeO}_{2}$ seed layer on plastic substrate for microelectronics applications. Thin Solid Films. 2004;453-454:100-105.

10. Kwon H, Hwang K, Park J, Ryu S, Kim SK. Electron solvation and solvation-induced crystallization of an ammonia film on $\mathrm{Ag}$ (111) studied by 2-photon photoemission. Physical Chemistry Chemical Physics. 2011;13(39):17785-17790.

11. Qi JQ, Guo R, Wang Y, Liu XW, Chan HLW. Electric FieldControlled Crystallizing $\mathrm{CaCO}_{3}$ Nanostructures from Solution. Nanoscale Research Letters. 2016;11:120.

12. Uda S, Koizumi H, Nozawa J, Fujiwara K. Crystal growth under external electric fields. In: International Conference of Computational Methods in Sciences and Engineering 2014 (ICCMSE 2014); 2014 Apr 4-7; Athens, Greece. AIP Conference Proceedings. 2014;1618;1:261-264.

13. Ahmed M, Arakel A, Hoey D, Thumarukudy MR, Goosen MF a, Al-Haddabi M, et al. Feasibility of salt production from inland RO desalination plant reject brine: A case study. Desalination. 2003;158(1-3):109-117.

14. Jönsson JÅ, Barri T. Supported-Liquid Membrane Extraction. In: Wilson ID, ed. Encyclopedia of Separation Science. Cambridge: Academic Press; 2000.

15. McCaffrey MA, Lazar B, Holland HD. The evaporation path of seawater and the coprecipitation of $\mathrm{Br}$ - and $\mathrm{K}+$ with halite. Journal of Sedimentary Petrology. 1987;57(5):928-938.

16. Mortimer RG. Physical Chemistry. Cambridge: Academic Press; 2000.

17. Revalor E, Hammadi Z, Astier JP, Grossier R, Garcia E, Hoff C, et al. Usual and unusual crystallization from solution. Journal of Crystal Growth. 2010;312(7):939-946.

18. Kurake N, Tanaka H, Ishikawa K, Nakamura K, Kajiyama H, Kikkawa F, et al. Synthesis of calcium oxalate crystals in culture medium irradiated with non-equilibrium atmospheric-pressure plasma. Applied Physics Express. 2016;9(9):096201.

19. Tendero C, Tixier C, Tristant P, Desmaison J, Leprince P. Atmospheric pressure plasmas: A review. Spectrochim Acta Part B: Atomic Spectroscopy. 2006;61(1):2-30.

20. Khlyustova A, Maksimov A. Double Electrical Layer at the Plasma-Solution Interface. Contributions to Plasma Physics. 2013;53(6):481-491.

21. Liu J, He B, Chen Q, Li J, Xiong Q, Yue G, et al. Direct synthesis of hydrogen peroxide from plasma-water interactions. Scientific Reports. 2016;6:38454

22. Navrátil Z, Trunec D, Šmíd R, Lazar L. A software for optical emission spectroscopy-problem formulation and application to plasma diagnostics. Czechoslovak Journal of Physics. 2006;56(Suppl 2):B944-B951.

23. Maksimov AI, Khlyustova AV. Influence of the composition of electrolyte on the kinetics of its nonequilibrium evaporation at the initial steps. High Energy Chemistry. 2009;43(1):51.

24. Sirotkin NA, Khlyustova AV, Maksimov AI. Numerical simulation of the gas phase composition in a glow discharge with an electrolyte cathode. Surface Engineering and Applied Electrochemistry. 2014;50(4):323-329.

25. Silberberg M. Principles of General Chemistry. New York: McGraw-Hill. 2009.

26. Warren JK. Evaporites: Sediments, Resources and Hydrocarbons. Berlin Heidelberg: Springer-Verlag; 2006.

27. Carpenter AB. Origin and Chemical Evolution of Brines in Sedimentary Basins. In: Society of Petroleum Engineers Annual Fall Technical Conference and Exhibition; 1978 Oct 1-3; Houston, TX, USA.

28. Barry PH, Lynch JW. Liquid junction potentials and small cell effects in patch-clamp analysis. The Journal of Membrane Biology. 1991;121(2):101-117.

29. Walker D, Verma PK, Cranswick LMD, Jones RL, Clark SM, Buhre S. Halite-sylvite thermoelasticity. American Mineralogist. 2004;89(1):204-210. 